\title{
Linkage analysis of smoking initiation and quantity in Dutch sibling pairs
}

JM Vink

AL Beem

D Posthuma

MC Neale

G Willemsen

KS Kendler

PE Slagboom

DI Boomsma

Correction to: The Pharmacogenomics Journal (2004) 4, 274-282. doi: 10.1038/sj.tpj.6500255

The Pharmacogenomics Journal (2004) 4, 345-346. doi:10.1038/sj.tpj.6500270

Following the publication of the above paper, the authors have identified an error in Figures 1 and 2 . The figures were switched accidentally and the authors would like to apologise for any confusion this might have caused. Figures 1 and 2 are reproduced correctly below.

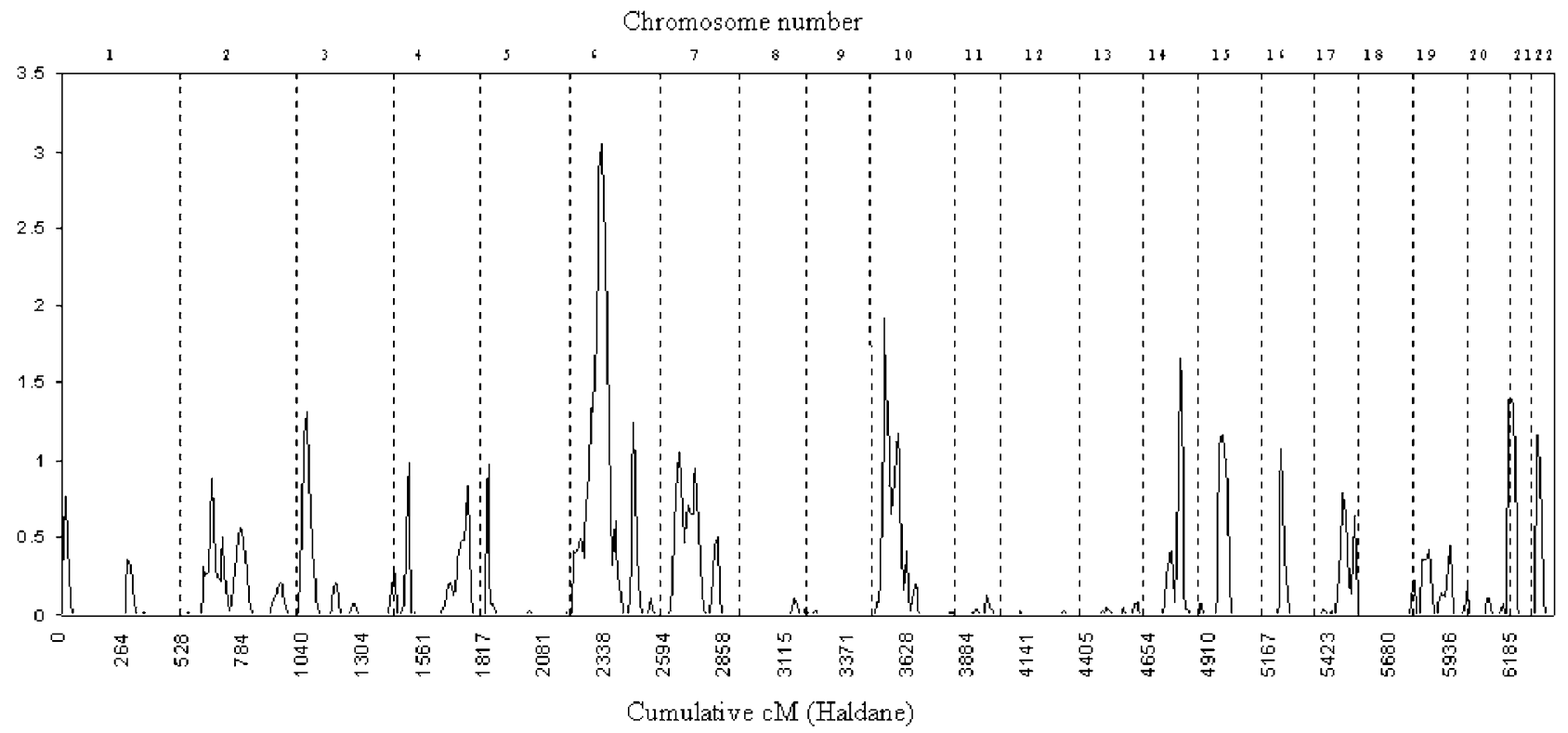

Figure 1 LOD scores across the genome for phenotype 'smoking initiation'. The cumulative Haldane centiMorgans are shown on the $x$-axis and LOD-score is shown on the $y$-axis. Chromosome number is shown at the top of the figure. 
neg

Corrigendum

346

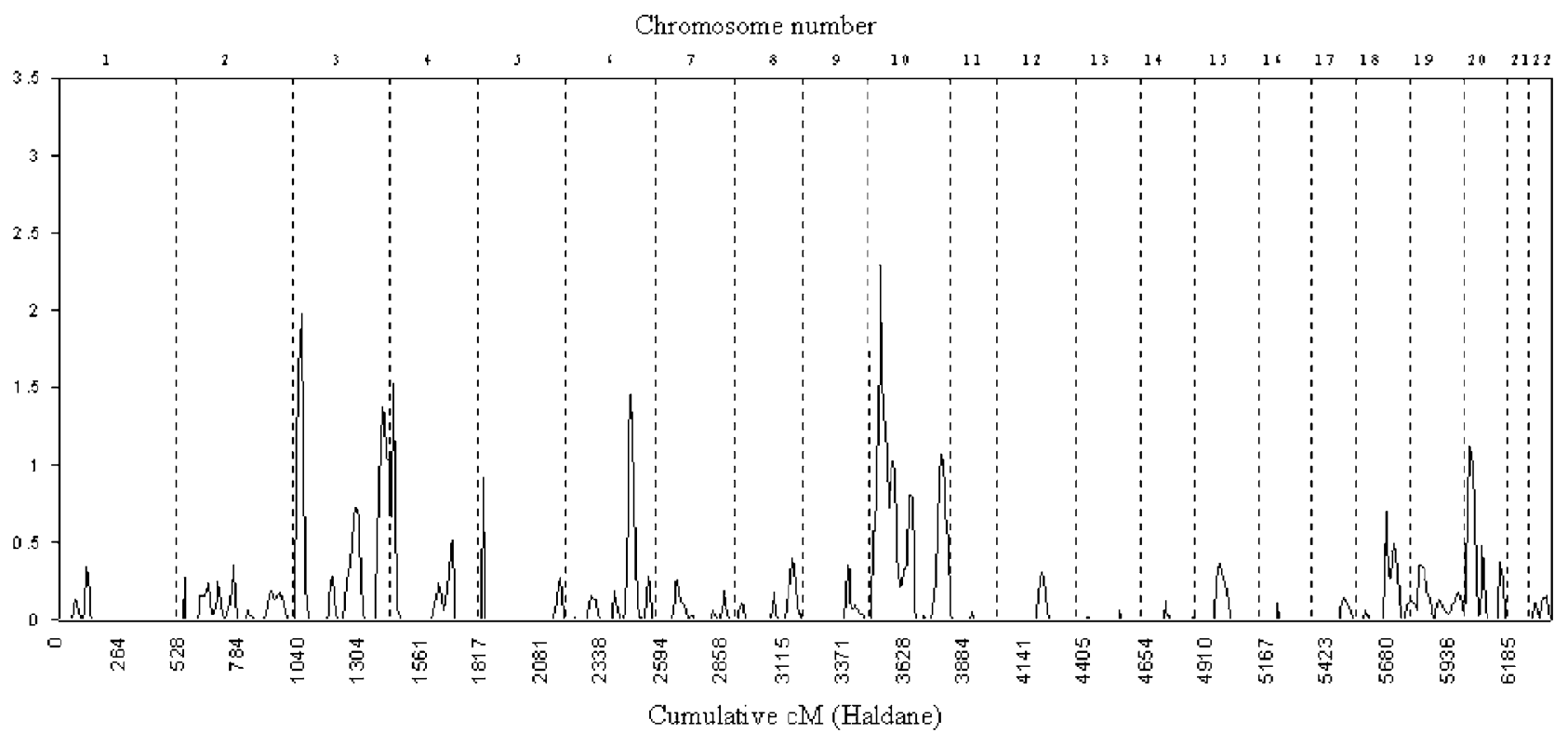

Figure 2 LOD scores across the genome for phenotype 'maximum number of cigarettes per day'. The cumulative Haldane centiMorgans are shown on the $x$-axis and LOD-score is shown on the $y$-axis. Chromosome number is shown at the top of the figure.

The Pharmacogenomics journal 